\title{
First principles study of magnetism induced by topological frustration of bowtie-shaped graphene nanoflake
}

\author{
Yang Ge a, ${ }^{\text {, Jianlong Ji }}{ }^{\mathrm{a}, 1}$, Zhizhong Shen ${ }^{\mathrm{a}}$, Qiang Zhang ${ }^{\mathrm{a}}$, Aoqun Jian ${ }^{\mathrm{a}}$, Qianqian Duan ${ }^{\mathrm{a}}$, \\ Chao Wang ${ }^{\mathrm{b}}$, Jun Jiang ${ }^{\mathrm{c}}$, Wendong Zhang ${ }^{\mathrm{a}}$, Shengbo Sang ${ }^{\mathrm{a}, *}$ \\ ${ }^{a}$ MicroNano System Research Center, Key Lab of Advanced Transducers and Intelligent Control System of the Ministry of Education \& College of Information \\ Engineering, Taiyuan University of Technology, Taiyuan 030024, China \\ ${ }^{\mathrm{b}}$ Institute of Mechanics, Chinese Academy of Sciences, Beijing 100190, China \\ ${ }^{\mathrm{c}}$ Beijing Computing Center, Beijing 100094, China
}

\section{A R T I C L E I N F O}

\section{Article history:}

Received 21 August 2017

Received in revised form

16 October 2017

Accepted 3 November 2017

Available online 3 November 2017

\begin{abstract}
A B S T R A C T
Fine-tuning magnetic states by understanding topological frustration inducing magnetic mechanism should allow greater flexibility for the design of graphene-based spintronics. Based on first-principles calculations, it is predicted that bowtie-shaped graphene nanoflake (GNF) is of spin-polarized ground state exhibiting antiferromagnetic (AFM) ordering between two individual triangular GNFs. It is demonstrated that strength of antiferromagnetic coupling of both symmetric and asymmetric bowtieshaped GNF displays strong zero-energy-state-orientated behavior due to non-trivial nature of topological frustration, with implications for designing graphene nanostructures with predefined magnetic states. It also proposes a specific example of structures that can serve as nanoscale molecular logic gates composed by asymmetric bowtie-shaped GNFs units, which augment the special antiferromagnetic function through structural configuration of multi-bowtie-shaped GNFs.
\end{abstract}

() 2017 Elsevier Ltd. All rights reserved.

\section{Introduction}

Spintronics, exploiting spin degree of freedom rather than charge of electron in conventional electronics, can achieve information storage, detection, logic, and communication capabilities on a single chip [1,2]. With the recent development of techniques like molecular beam epitaxy (MBE) [3], it becomes possible to fabricate multilayers or even monolayer molecular spintronics [4,5]. Graphene, easily tailored than one-dimensional nanomaterials for planar geometry at the atomic-size scale, such as nanowires (NWs) and carbon nanotubes (CNTs) [6], holds the promise as a spin channel material for its room temperature physical properties including long spin lifetimes and spin diffusion lengths due to low intrinsic spin-orbit and hyperfine coupling in carbon [6-12]. Recently, twin effects of quantum valley Hall and quantum spin Hall could be probed simultaneously in graphene and will significantly advance the field of graphene spintronics, hitherto hobbled by the lack of spin-orbit interaction [13].

\footnotetext{
* Corresponding author.

E-mail address: sunboa-sang@tyut.edu.cn (S. Sang).

1 These authors contributed equally to this work.
}

However, perfect graphene's nonmagnetic ground state impedes its application in spintronics [14]. When a graphene sheet is cut into graphene nanoflake (also referred to as nanodot, nanoisland or nanodisk), the newly generated C-dangling bonds at the edge cause localized edge states (nonbonding $\pi$-electron state) along the high-symmetry zigzag direction or along any lowsymmetry (chiral) direction boundaries [15], which has been verified experimentally [16-18]. Furthermore, due to the large contribution to density of states at Fermi energy by localized edge states, remarkable electron-electron correlation gives rise to localized spins that bring about an intriguing carbon-only edge magnetism favoring applications in graphene-based spintronics [14,19-21]. Also, important effects of edges on magnetism and the spindependent transport properties of symmetric and asymmetric zigzag-edged graphene nanoribbon (ZGNR) have been demonstrated [22]. Recently, magnetic orderings on graphene edges in controlled zigzag orientation have been confirmed stable even at room temperature experimentally, raising hopes of graphene nanoflake as the most favorable candidate material in future molecular spintronics/electronics devices operating under ambient conditions $[23,24]$. Encouragingly, substantial progress of precise nanofabrication techniques and bottom-up synthesis for 
preparation of graphene nanostructures indeed make it possible to produce specific well-defined atomically precise zigzag edge and triangular shapes with tunable size [25-32].

Building graphene-based spintronics with predetermined magnetic properties has long been the focus of basic and applied research. Edge states of graphene nanoflake from the resulting geometrical frustration are further topologically characterized. Contrary to its implication of the name, frustrated systems actually have more configurational options than conventional collective systems [33]. Basic concepts of frustrated systems are turning out to have more practical uses, and geometrical frustration can manifest in well-defined structures [34-36]. It has been concluded that the appearance of magnetism requires presence of defects, since the presence of defects can introduce important changes in electronic structure of graphene, leading to phenomena such as carbon magnetism [37]. A distinct origin of this unconventional carbononly magnetism in finite graphene structures is the topological frustration of $\pi$-bonds, first introduced by Wang who has made a rigorous classification depending on whether only one or both sublattices of graphitic structure are frustrated for arbitrarilyshaped graphene nanoflake [38]. Typical representative shape of the second class with both sublattices topologically frustrated is zigzag bowtie-shaped graphene nanoflake (GNF), also known as Clar's goblet, composed of two zigzag-edged triangle fragments sharing hexagons, as shown in Fig. 1(a). In particular, a zigzagedged triangle with one sublattice topologically frustrated has nonzero net magnetization but scales linearly with fragment size [39-41]. With this property, bowtie-shaped GNF may have antiferromagnetic (AFM) ordering by connecting two individual triangular GNFs, acting as a fundamental single-molecule NOT logic gate.

Following the study on magnetic feature of bowtie-shaped GNF, there have been several related reports on the magnetic states of hydrogenated diamond-shaped zigzag graphene quantum dots, intrinsic ferromagnetism in two-dimensional (2D) carbon semiconducting structures, magnetic-field control of magnetism in bowtie-shaped GNF and alternative spintronic designs [42-45]. These studies all concluded that magnetic properties of GNF are of strongly geometry dependence. However, the underlying topological frustration inducing mechanism that is prerequisite in predicting the strength of magnetic coupling of specific GNF hasn't been given out. Thus, it is the purpose of our paper to explore the underlying topological frustration inducing mechanism with a detailed analysis of the effect of zero energy state on the magnetic properties of symmetric and asymmetric (also can be referred to axisymmetric and centrosymmetric) bowtie-shaped GNFs. Analytical models with varying structure configurations from the

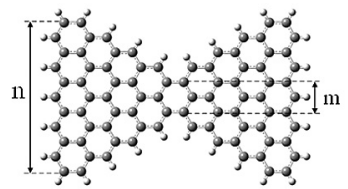

(a)

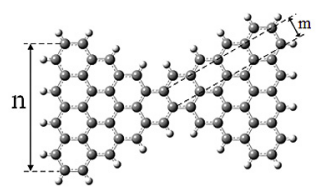

(d)

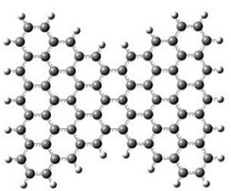

(b)

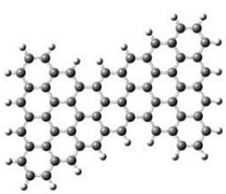

(e)

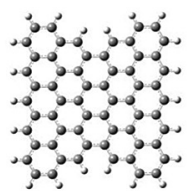

(c)

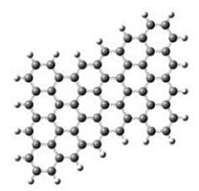

(f)
Fig. 1. The two sets of representative symmetric and asymmetric bowtie-shaped GNFs. (a) n5m1, (b) n5m2, (c) n5m3, (d) n4m1, (e) n4m2, (f) n4m3. perspective of topological frustration were developed to demonstrate a general controlled zero-energy-state-orientated magnetic behavior on spintronics employing first-principles calculations. The work provides explicitly a nonmonotonic relationship between the number of zero-energy-state and the strength of antiferromagnetic coupling for bowtie-shaped GNF, unveiling an underlying topological frustration inducing mechanism: manipulation on the number of zero energy state. Furthermore, a specific example of nanostructured logic gate composing of three asymmetric bowtieshaped GNF units is presented.

\section{Method}

The proposed symmetric and asymmetric hydrogenated bowtie-shaped graphene nanoflake is composed of two individual triangular fragment with different triangle side length $\mathrm{n}$ and connecting width $\mathrm{m}$, where $\mathrm{n}=3,4, \ldots, 7$, and the connecting width $\mathrm{m}=1,2,3,4, \ldots$ Two sets of representative symmetric and asymmetric bowtie-shaped GNF are shown in Fig. 1, n5m1, n5m2, n5m3, $\mathrm{n} 4 \mathrm{~m} 1, \mathrm{n} 4 \mathrm{~m} 2, \mathrm{n} 4 \mathrm{~m} 3$. All calculations on geometry optimizations were carried out based on first-principles Density functional Theory (DFT) using Gaussian 09 program package [46]. Spin-polarized calculation was obtained using the functional of the Perdew-BurkeErnzerh (PBE) [47] realization of the generalized gradient approximation and $6-31 G^{* *}$ Gaussian basis set. The initial bond length between all carbon atoms is set to $1.42 \AA$ A. Calculations of such openshell singlet ground states are not always trivial since the system has equal numbers of spin up and spin down electrons that require explicit spatial spin-symmetry breaking, and merely using an unrestricted method will fail, leading to higher ground state energy. Therefore, fragment molecular orbital method [48-50] was adopted to get an initial spin-symmetry guess wave functions, and then such a broken spin-symmetry initial wave function was used to get singlet ground states for symmetric and asymmetric bowtieshaped GNF with zero net spin.

\section{Results and disscussion}

First, a large set of bowtie-shaped nanoflakes of different triangle size $\mathrm{n}$ and connecting width $\mathrm{m}$ for both symmetric and asymmetric geometries was calculated, and some representative examples are shown in Fig. 1. In Fig. 2 (a) and (b), spin density of representative structures of symmetric and asymmetric configuration of ground state are shown using the PBE density functional. In Fig. 2, different colors indicate different spin states: the color blue represents the spin-up magnetic state while the red corresponds to the spin-down magnetic state. The two connected triangles show opposite net spins, namely, the left triangle shows net

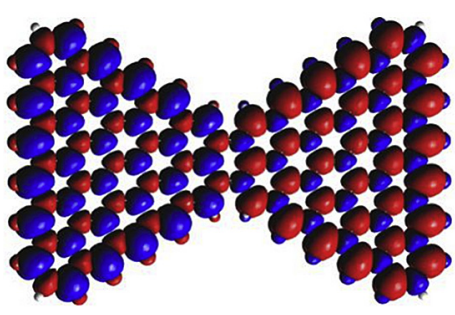

(a)

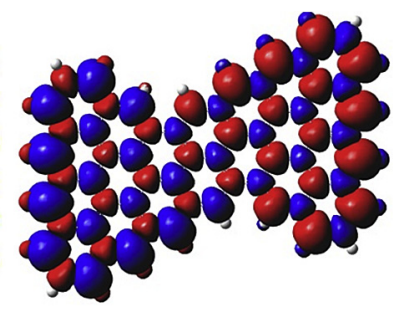

(b)
Fig. 2. The spin density distributions of the singlet ground state of symmetric and asymmetric bowtie-shaped (a) n6m1, (b) $n 4 \mathrm{~m} 2$ GNFs obtained using the spinunrestricted (U) PBE/6-31G** method. Different colors indicate different spin states: the color blue represents the spin-up magnetic state while red corresponds to the spin-down magnetic state. (A colour version of this figure can be viewed online.) 
spin-down magnetic state, and the right triangle shows net spin-up magnetic state. Therefore, the structures studied here give rise to a strong antiferromagnetic (AFM) coupling, as shown in Fig. 2. All depicted structures exhibit open-shell spin-polarized singlet ground state that magnetic moments localized at two individual triangles and oriented antiparallel in both halves of the nanostructure. Since such structures have equal number of carbon atoms in the two sublattices A and B, according to Lieb's theorem, they have no net magnetization, and Hund's rule breaks down. This is a remarkable finding that there exists magnetic orderings even for GNF with balanced sublattices. In other words, it is the ground electronic configuration that breaks the spin-spatial symmetry and exhibits antiferromagnetic ordering, as shown in Fig. 2. The structure presenting strong antiferromagnetic features is a natural NOT logic gate, since flipping the input spin in one side of bowtie shape requires the output spins to flip on the other side as well, enabling practically accessible logic operations [38].

Furthermore, the ground state of bowtie-shaped GNF from the frontier molecular orbital (FMO) prospect was studied and explained in terms of localization. In Fig. 3, red and green colors indicate positive and negative phases of wave function whose square represents electron cloud density. It is clear that the wave functions of spin-up highest occupied molecular orbital ( $\alpha$-HOMO) shown in Fig. 3(a) and spin-down highest occupied molecular orbital ( $\beta$-HOMO) Fig. 3(b) exhibit identical phase characteristics, but were completely localized on opposite triangles rather than distributing over the whole bowtie-shaped sample. Therefore, the spreading of electron cloud density on opposite triangles strongly exhibits the antiferromagnetic coupling characteristic of electrons, in agreement with previous calculations on several singly occupied orbitals of a representative bow-tie GNF [38]. Similarly, wave functions of the spin-up lowest unoccupied molecular orbital $(\alpha-$ LUMO) shown in Fig. 3(c) and spin-down lowest unoccupied molecular orbital ( $\beta$-LUMO) shown in Fig. 3(d) also demonstrate the same antiferromagnetic coupling features. Therefore, the openshell singlet nature of the system is qualitatively illustrated for these bowtie-shaped GNFs.

Next, we consider the most important relationship between zero-energy states and the strength of magnetic coupling for both symmetric and asymmetric geometries, quantitatively. According to the counting rule referred in Ref. [40], the number of zero-energy states of structures referred as nullity equals to $2^{*}(2 \alpha-N)$, where $\mathrm{N}$

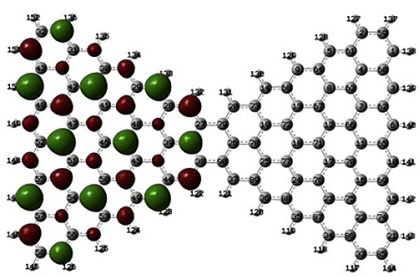

(a) Spin-up HOMO

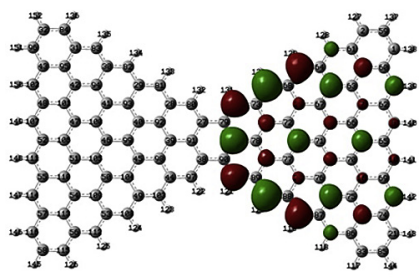

(c) Spin-up LUMO

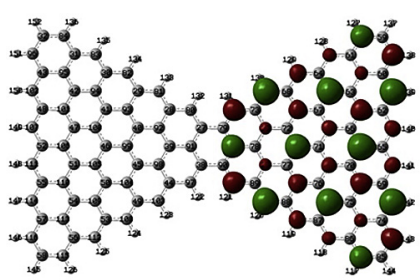

(b) Spin-down HOMO

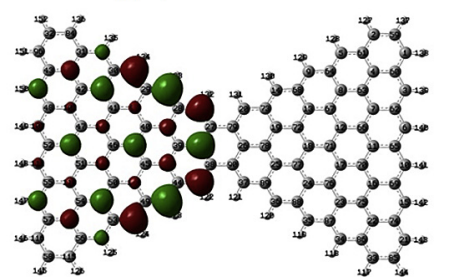

(d) Spin-down LUMO
Fig. 3. The spin-up and spin-down polarized HOMO and LUMO of the ground state of a typical symmetric bowtie-shaped GNF for $\mathrm{n} 6 \mathrm{~m} 1$, obtained using the spin-unrestricted (U) $\mathrm{PBE} / 6-31 \mathrm{G}^{* *}$ method. (A colour version of this figure can be viewed online.) is the total number of sites and $\alpha$ is the maximum possible number of non-adjacent sites involving atoms belonging to both sublattices $A$ and $B$ in bowtie-shaped GNF. The strength of magnetic coupling is the energy difference between ferromagnetic (FM) and antiferromagnetic (AFM) configurations. For these graphene nanoflakes, the following spin states: spin-polarized (open-shell) singlet state and spin polarized triplet state (open-shell) were obtained, with total energies are $\mathrm{E}_{\mathrm{AFM}}$ and $\mathrm{E}_{\mathrm{FM}}$, respectively.

The calculated results of nullity and strength of magnetic coupling for both symmetric and asymmetric bowtie-shaped structures with varying triangle size $\mathrm{n}$ and connecting width $\mathrm{m}$ are shown in Table 1 . The number of zero energy states for symmetric and asymmetric bowtie-shaped GNF is $2^{*}(n-m-1)$ in either case. As shown in Table 1 , no matter symmetric or asymmetric bowtie-shaped GNF, the strength of magnetic coupling drops with the decrease of the number of nullity for the same triangle size $n$. For instance, symmetric bowtie-shaped GNFs of $n 6 m 1, n 6 m 2$, or n6m3 with the nullity of 8,6 , and 4 , respectively, have decreasing magnetic strength of $245 \mathrm{meV}, 205 \mathrm{meV}$ and $165 \mathrm{meV}$, respectively. However, it is hard to find trends for bowtie-shaped GNF with equal number of nullity, since equal number of nullity can be induced by topological frustration from different triangle sizes and connecting widths. In other words, is has to confirm either triangle size or connecting width. To verify this assumption, the strength of magnetic coupling with respect to the same connecting width $\mathrm{m}$ was studied. In Fig. 4, the magnetic coupling strength as a function of triangular size with the same connecting widths of symmetric and asymmetric bowtie-shaped GNF is presented for the three sets. Unexpectedly, the plots clearly show that the tendency of magnetic coupling strength does not increase monotonously with the size of the triangle. This variation has two implications: (i) the strength of antiferromagnetic coupling can be modulated by the nullity induced by topological frustration, (ii) size effect in quantum system diminishes the minimum energy level splitting, leading to a decrease of antiferromagnetic coupling strength. Therefore, with an increase of triangle size or nullity, the coupling strength of all three representative sets of different widths experiences an increase before reaching a maximum, then decrease to convergence, as shown in Fig. 4. Furthermore, energy difference between ferromagnetic and antiferromagnetic coupling obtained were all greater than thermodynamic threshold $18 \mathrm{meV}$, which can make the structure achieve robust operation at reasonable temperatures [51].

To further clarify the logic function, three-terminal NOR and NAND gates were studied, in which the central triangle is connected to three other triangles just like the previously described three connected asymmetric bowtie-shaped GNFs. In this illustrating structure shown in Fig. 5 (a), the region is separated into A, $B, C$ and D, respectively. It is clearly shown in Fig. 5 (b), (c) and (d) that these tend to be AFM coupled. Regions A and $B$ are set as input operands; $C$ is programming bit; $D$ is output. Spin up state 1 is defined as Boolean true and spin down state 0 as Boolean false. A NAND or NOR logic gate can be made by setting 1 or 0 to $C$. For the first case, set $C$ is set as $0, A$ and $B$ as input are assigned for 1 and 0 , respectively, then we get a NAND gate for $\mathrm{D}=\overline{A \cap B}=1$, shown in red in Fig. 5(b). For the second case, set $\mathrm{C}$ is set as $1, \mathrm{~A}$ and $\mathrm{B}$ as input are assigned for 1 and 0 , respectively, then a NOR gate for $D=\overline{A \cup B}=0$ is obtained, shown in blue in Fig. 5(c). For another case, set $C$ is set as $1, A$ and $B$ as input are assigned for 1 and 1, respectively, then a NOR gate for $\mathrm{D}=\overline{A \cup B}=0$ is obtained, shown in blue in Fig. 5(d).

These above three logic cases induced by special structure configuration present a typical Boolean logic designation that employs spin degree of freedom instead of charge of electron used in conventional electronics. The coupling strength for the above three cases well outnumbered the thermodynamic threshold $18 \mathrm{meV}$ 
Table 1

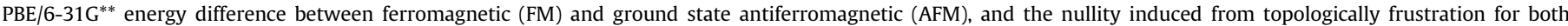
symmetric and asymmetric bowtie-shape structure.

\begin{tabular}{|c|c|c|c|c|c|}
\hline Symmetric bowtie-shaped & Nullity & $\mathrm{E}_{\mathrm{FM}}-\mathrm{E}_{\mathrm{AFM}}(\mathrm{meV})$ & Asymmetric bowtie-shaped & Nullity & $\mathrm{E}_{\mathrm{FM}}-\mathrm{E}_{\mathrm{AFM}}(\mathrm{meV})$ \\
\hline$n 3 m 1$ & 2 & 18 & $\mathrm{n} 3 \mathrm{~m} 1$ & 2 & 88 \\
\hline $\mathrm{n} 4 \mathrm{~m} 1$ & 4 & 155 & $\mathrm{n} 4 \mathrm{~m} 1$ & 4 & 137 \\
\hline $\mathrm{n} 4 \mathrm{~m} 2$ & 2 & 18 & $\mathrm{n} 4 \mathrm{~m} 2$ & 2 & 84 \\
\hline- & - & - & $\mathrm{n} 4 \mathrm{~m} 3$ & 0 & 33 \\
\hline $\mathrm{n} 5 \mathrm{~m} 1$ & 6 & 194 & $\mathrm{n} 5 \mathrm{~m} 1$ & 6 & 171 \\
\hline $\mathrm{n} 5 \mathrm{~m} 2$ & 4 & 127 & $\mathrm{n} 5 \mathrm{~m} 2$ & 4 & 153 \\
\hline $\mathrm{n} 5 \mathrm{~m} 3$ & 2 & 43 & $\mathrm{n} 5 \mathrm{~m} 3$ & 2 & 128 \\
\hline $\mathrm{n} 6 \mathrm{~m} 1$ & 8 & 245 & $\mathrm{n} 6 \mathrm{~m} 1$ & 8 & 346 \\
\hline $\mathrm{n} 6 \mathrm{~m} 2$ & 6 & 205 & $\mathrm{n} 6 \mathrm{~m} 2$ & 6 & 210 \\
\hline n6m3 & 4 & 165 & $\mathrm{n} 6 \mathrm{~m} 3$ & 4 & 183 \\
\hline $\mathrm{n} 7 \mathrm{~m} 1$ & 10 & 320 & $\mathrm{n} 7 \mathrm{~m} 1$ & 10 & 416 \\
\hline $\mathrm{n} 7 \mathrm{~m} 2$ & 8 & 260 & $\mathrm{n} 7 \mathrm{~m} 2$ & 8 & 355 \\
\hline $\mathrm{n} 7 \mathrm{~m} 3$ & 6 & 142 & $\mathrm{n} 7 \mathrm{~m} 3$ & 6 & 270 \\
\hline $\mathrm{n} 8 \mathrm{~m} 1$ & 12 & 264 & $\mathrm{n} 8 \mathrm{~m} 1$ & 12 & 361 \\
\hline $\mathrm{n} 8 \mathrm{~m} 2$ & 10 & 232 & $\mathrm{n} 8 \mathrm{~m} 2$ & 10 & 324 \\
\hline $\mathrm{n} 8 \mathrm{~m} 3$ & 8 & 122 & $\mathrm{n} 8 \mathrm{~m} 3$ & 8 & 255 \\
\hline
\end{tabular}
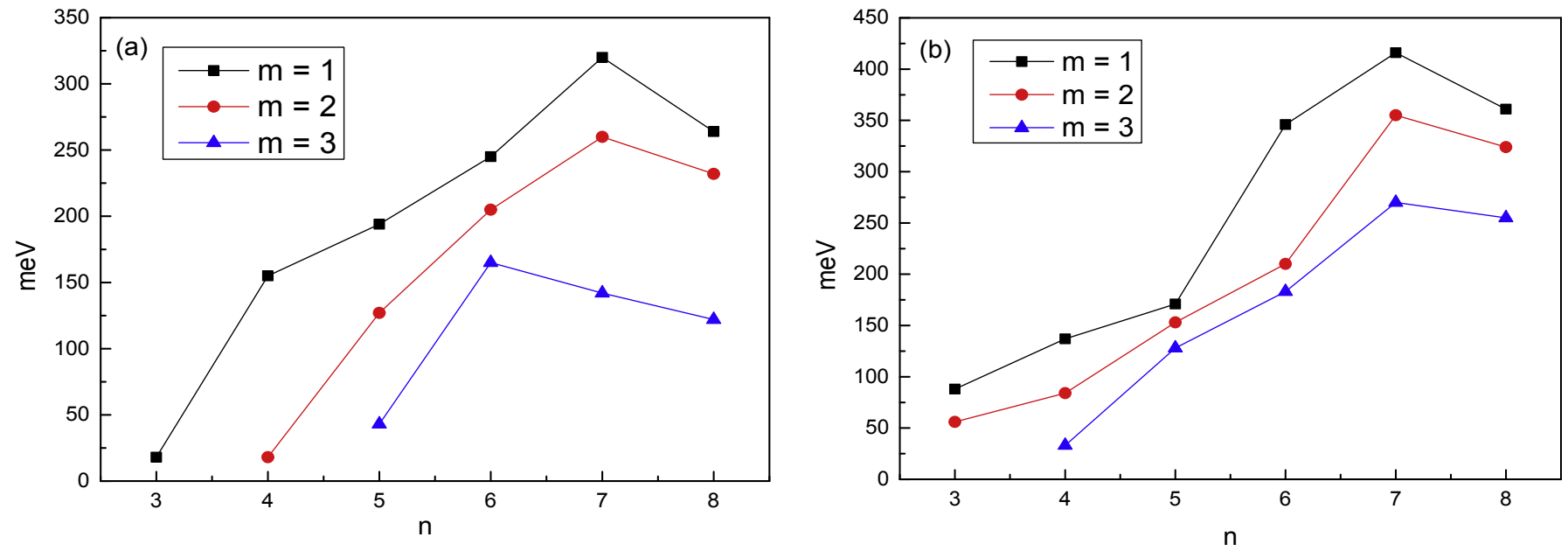

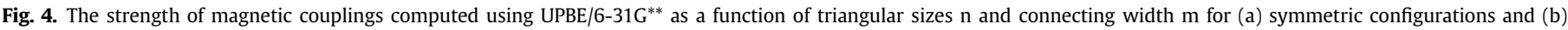
asymmetric configurations. (A colour version of this figure can be viewed online.)

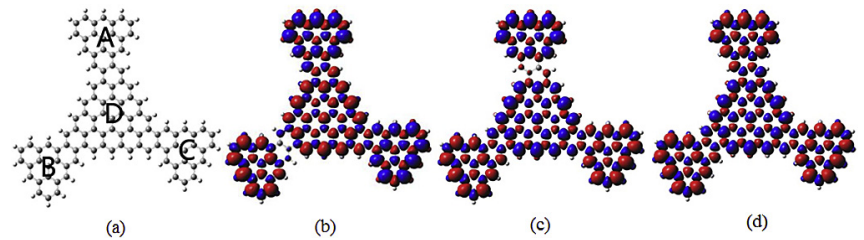

Fig. 5. Programmable logic gate structure and spin density distributions of the structure configuration (a) asymmetrical bowtie-shaped GNF units structure (b) spin NAND gate, (c) spin NOR gate obtained using the spin-unrestricted (U) PBE/6-31G** method. (A colour version of this figure can be viewed online.)

[51], which confirms that the structure can maintain spintronic properties for this programmable logic gate. The truth table for the above three-terminal logic gate shown in Table 2 enumerates over all logical combinations of all inputs and possible outputs. In addition, the structure configuration can be designed using different combinations of triangle unit, either symmetric or asymmetric bowtie-shaped GNF. This is consistent with results from a previous study of fundamental (NOR and NAND) logic gate composed by symmetric bowtie-shaped GNF and diamond-shaped zigzag graphene quantum dot (GNF) $[38,42]$.
Table 2

The truth logic table for the above three-terminal logic gate.

\begin{tabular}{lllll}
\hline Gate & A & B & C & D \\
\hline NOR & 0 & 0 & 0 & 1 \\
NOR & 0 & 1 & 0 & 0 \\
NOR & 1 & 0 & 0 & 0 \\
NOR & 1 & 1 & 0 & 0 \\
NAND & 0 & 0 & 1 & 1 \\
NAND & 0 & 1 & 1 & 1 \\
NAND & 1 & 0 & 1 & 1 \\
NAND & 1 & 1 & 1 & 0
\end{tabular}

\section{Conclusion}

In this paper, the PBE results of a first-principles study of topological frustration effect on magnetic property of bowtie-shaped GNF were reported. Our findings unambiguously demonstrate that both symmetric and asymmetric bowtie-shaped GNF display strongly nullity-orientated magnetic behavior induced by topological frustration. This described relationship opens a way toward designing graphene nanostructures with predefined magnetic states from the structure-property prospective, a highly useful tool for the design and development of novel spintronic devices. From a 
technological perspective, this is a significant finding, since characterization of magnetic properties on the structure configuration of the GNF is a key prerequisite for device engineering.

\section{Acknowledgments}

We would also like to thank Gaokeng Xiao for Gaussian software supporting by Guangzhou Molcalx Information \& Technology Ltd, Professor Li Ming in Taiyuan University of Technology for providing calculation resource and China Lvliang Cloud Computing Center for calculation platform supporting.

This work is supported by the 863 project No. 2015AA042601, National Science Foundation of China Nos. 51705354, 61471255, 61474079, 61501316, 51505324 and 51622507, the Basic Research Project of Shanxi Province No.2015021092 and the Graduate student education innovation project of Shanxi (Grant No. 2017SY062).

\section{References}

[1] S.A. Wolf, D.D. Awschalom, R.A. Buhrman, J.M. Daughton, S.V. Molnár, M.L. Roukes, et al., Spintronics: a spin-based electronics vision for the future, Science 294 (5546) (2001) 1488-1495.

[2] A. Fert, Nobel Lecture: origin, development, and future of spintronics, Rev. Mod. Phys. 80 (4) (2008) 1517-1530.

[3] C.-L. Song, Y.-L. Wang, Y.-P. Jiang, Y. Zhang, C.-Z. Chang, L. Wang, et al., Topological insulator Bi2Se3 thin films grown on double-layer graphene by molecular beam epitaxy, Appl. Phys. Lett. 97 (14) (2010), 143118.

[4] M. Ganzhorn, W. Wernsdorfer, Molecular spintronics using single-molecule magnets, Nat. Mater. 7 (3) (2008) 179-186.

[5] I.V. Pinchuk, P.M. Odenthal, A.S. Ahmed, W. Amamou, J.E. Goldberger, R.K. Kawakami, Epitaxial co-deposition growth of CaGe2 films by molecular beam epitaxy for large area germanane, J. Mater. Res. 29 (03) (2014) 410-416.

[6] J. Ji, Z. Zhou, X. Yang, W. Zhang, S. Sang, P. Li, Establishment of onedimensional nano-interconnections, Small 9 (18) (2013) 3014-3029.

[7] D. Huertas-Hernando, F. Guinea, A. Brataas, Spin-orbit coupling in curved graphene, fullerenes, nanotubes, and nanotube caps, Phys. Rev. B 74 (15) (2006), 155426.

[8] N. Tombros, C. Jozsa, M. Popinciuc, H.T. Jonkman, B.J. van Wees, Electronic spin transport and spin precession in single graphene layers at room temperature, Nature 448 (7153) (2007) 571-574.

[9] B. Trauzettel, D.V. Bulaev, D. Loss, G. Burkard, Spin qubits in graphene quantum dots, Nat. Phys. 3 (3) (2007) 192-196.

[10] D. Huertas-Hernando, F. Guinea, A. Brataas, Spin-orbit-mediated spin relaxation in graphene, Phys. Rev. Lett. 103 (14) (2009), 146801.

[11] W. Han, R.K. Kawakami, Spin relaxation in single-layer and bilayer graphene, Phys. Rev. Lett. 107 (4) (2011), 047207.

[12] W. Han, R.K. Kawakami, M. Gmitra, J. Fabian, Graphene spintronics, Nat. Nanotechnol. 9 (10) (2014) 794-807.

[13] S.K.F. Islam, C. Benjamin, A scheme to realize the quantum spin-valley Hall effect in monolayer graphene, Carbon 110 (2016) 304-312.

[14] Y. Shibayama, H. Sato, T. Enoki, Disordered magnetism at the metal-insulator threshold in nano-graphite-based carbon materials, Phys. Rev. Lett. 84 (8) (2000) 1744-1747.

[15] T. Enoki, S. Fujii, K. Takai, Zigzag and armchair edges in graphene, Carbon 50 (9) (2012) 3141-3145

[16] Y. Kobayashi, K.-I. Fukui, T. Enoki, K. Kusakabe, Edge state on hydrogenterminated graphite edges investigated by scanning tunneling microscopy, Phys. Rev. B 73 (12) (2006), 125415

[17] Y. Niimi, T. Matsui, H. Kambara, K. Tagami, M. Tsukada, H. Fukuyama, Scanning tunneling microscopy and spectroscopy of the electronic local density of states of graphite surfaces near monoatomic step edges, Phys. Rev. B 73 (8) (2006), 085421.

[18] K.A. Ritter, J.W. Lyding, The influence of edge structure on the electronic properties of graphene quantum dots and nanoribbons, Nat. Mater. 8 (3) (2009) 235-242.

[19] K. Nakada, M. Fujita, Edge state in graphene ribbons Nanometer size effect and edge shape dependence, Phys. Rev. B Condens. Matter 54 (24) (1996) 17954-17961.

[20] Y. Miyamoto, K. Nakada, M. Fujita, First-principles study of edge states of Hterminated graphitic ribbons, Phys. Rev. B 59 (15) (1999) 9858-9861.

[21] K. Kusakabe, M. Maruyama, Magnetic nanographite, Phys. Rev. B 67 (9) (2003) $552-555$.

[22] X.Q. Deng, Z.H. Zhang, G.P. Tang, Z.Q. Fan, C.H. Yang, Spin filter effects in zigzag-edge graphene nanoribbons with symmetric and asymmetric edge hydrogenations, Carbon 66 (2) (2014) 646-653.

[23] T. Enoki, Y. Kobayashi, K.-I. Fukui, Electronic structures of graphene edges and nanographene, Int. Rev. Phys. Chem. 26 (4) (2007) 609-645.

[24] G.Z. Magda, X. Jin, I. Hagymási, P. Vancsó, Z. Osváth, P. Nemes-Incze, et al.,
Room-temperature magnetic order on zigzag edges of narrow graphene nanoribbons, Nature 514 (7524) (2014) 608-611.

[25] L.C. Campos, V.R. Manfrinato, J.D. Sanchez-Yamagishi, J. Kong, P. Jarillo-Herrero, Anisotropic etching and nanoribbon formation in single-layer graphene, Nano Lett. 9 (7) (2009) 2600-2604.

[26] L. Ci, L. Song, D. Jariwala, A.L. ElÃ-as, W. Gao, M. Terrones, et al., Graphene shape control by multistage cutting and transfer, Adv. Mater. 21 (44) (2009) 4487-4491.

[27] X. Jia, M. Hofmann, V. Meunier, B.G. Sumpter, J. Campos-Delgado, J.M. RomoHerrera, et al., Controlled formation of sharp zigzag and armchair edges in graphitic nanoribbons, Science 323 (5922) (2009) 1701-1705.

[28] F. Schäffel, J.H. Warner, A. Bachmatiuk, B. Rellinghaus, B. Büchner, L. Schultz et al., Shedding light on the crystallographic etching of multi-layer graphene at the atomic scale, Nano Res. 2 (9) (2009) 695-705.

[29] X. Yan, X. Cui, L.-S. Li, Synthesis of large, stable colloidal graphene quantum dots with tunable size, J. Am. Chem. Soc. 132 (17) (2010) 5944-5945.

[30] X. Zhang, O.V. Yazyev, J. Feng, L. Xie, C. Tao, Y.-C. Chen, et al., Experimentally engineering the edge termination of graphene nanoribbons, Acs Nano 7 (1) (2013) 198-202.

[31] C.K. Chua, Z. Sofe, P. Simek, O. Jankovsky, K. Klımova, S. Bakardjieva, et al, Synthesis of strongly fluorescent graphene quantum dots by cage-opening buckminsterfullerene, Acs Nano 9 (3) (2015) 2548-2555.

[32] P. Ruffieux, S. Wang, B. Yang, C. Sanchez-Sanchez, J. Liu, T. Dienel, et al., Onsurface synthesis of graphene nanoribbons with zigzag edge topology, Nature 531 (7595) (2016) 489-492.

[33] A.P. Ramirez, Geometric frustration: magic moments, Nature 421 (30) (2003) 483.

[34] A.J. Pak, E. Paek, G.S. Hwang, Tailoring the performance of graphene-based supercapacitors using topological defects: a theoretical assessment, Carbon 68 (13) (2014) 734-741.

[35] Y. Nahas, S. Prokhorenko, L. Bellaiche, Frustration and self-ordering of topological defects in ferroelectrics, Phys. Rev. Lett. 116 (11) (2016), 117603.

[36] J. Drisko, T. Marsh, J. Cumings, Topological frustration of artificial spin ice, Nat. Commun. 8 (17) (2017) 14009.

[37] T.P. Kaloni, M. Upadhyay Kahaly, R. Faccio, U. Schwingenschlögl, Modelling magnetism of C at O and B monovacancies in graphene, Carbon 64 (9) (2013) $281-287$

[38] W.L. Wang, O.V. Yazyev, S. Meng, E. Kaxiras, Topological frustration in graphene nanoflakes: magnetic order and spin logic devices, Phys. Rev. Lett. 102 (15) (2009), 157201.

[39] J. Fernández-Rossier, J.J. Palacios, Magnetism in graphene nano-islands, Phys. Rev. Lett. 99 (17) (2007), 177204.

[40] W.L. Wang, S. Meng, E. Kaxiras, Graphene NanoFlakes with large spin, Nano Lett. 8 (1) (2008) 241-245.

[41] O. Voznyy, A.D. Güçlü, P. Potasz, P. Hawrylak, Effect of edge reconstruction and passivation on zero-energy states and magnetism in triangular graphene quantum dots with zigzag edges, Phys. Rev. B 83 (16) (2011), 165417.

[42] L.A. Agapito, N. Kioussis, E. Kaxiras, Electric-field control of magnetism in graphene quantum dots: ab initio calculations, Phys. Rev. B Condens. Matter Mater. Phys. 82 (20) (2010), 201411.

[43] J. Zhou, Q. Wang, Q. Sun, P. Jena, Intrinsic ferromagnetism in two-dimensional carbon structures: triangular graphene nanoflakes linked by carbon chains, Phys. Rev. B 84 (8) (2011), 081402.

[44] K. Szałowski, Ground-state magnetic phase diagram of bow-tie graphene nanoflakes in external magnetic field, J. Appl. Phys. 114 (24) (2013), 243908.

[45] Z. Bullard, E.C. Girao, J.R. Owens, W.A. Shelton, V. Meunier, Improved allcarbon spintronic device design, Sci. Rep. 5 (2015) 7634.

[46] M.J. Frisch, G.W. Trucks, H.B. Schlegel, G.E. Scuseria, M.A. Robb, J.R. Cheeseman, G. Scalmani, V. Barone, B. Mennucci, G.A. Petersson, H. Nakatsuji, M. Caricato, X. Li, H.P. Hratchian, A. F. Izmaylov, J. Bloino, G. Zheng, J.L. Sonnenberg, M. Hada, M. Ehara, K. Toyota, R. Fukuda, J. Hasegawa, M. Ishida, T. Nakajima, Y. Honda, O. Kitao, H. Nakai, T. Vreven, J.A. Montgomery Jr., J.E. Peralta, F. Ogliaro, M. Bearpark, J.J. Heyd, E. Brothers, K.N. Kudin, V.N. Staroverov, R. Kobayashi, J. Normand, K. Raghavachari, A. Rendell, J.C. Burant, S.S Iyengar, J. Tomasi, M. Cossi, N. Rega, J.M. Millam, M. Klene, J.E. Knox, J.B. Cross, V. Bakken, C. Adamo, J. Jaramillo, R. Gomperts, R.E. Stratmann, O. Yazyev, A.J Austin, R. Cammi, C. Pomelli, J.W. Ochterski, R.L. Martin, K. Morokuma, V.G. Zakrzewski, G.A. Voth, P. Salvador, J.J. Dannenberg, S. Dapprich, A.D. Daniels, Ö. Farkas, J.B. Foresman, J.V. Ortiz, J. Cioslowski, D.J. Fox, Gaussian, Inc Wallingford, CT, 2009.

[47] J. Perdew, K. Burke, M. Ernzerhof, Generalized gradient approximation made simple, Phys. Rev. Lett. 77 (18) (1996) 3865.

[48] Y. Inadomi, T. Nakano, K. Kitaura, U. Nagashima, Definition of molecular orbitals in fragment molecular orbital method, Chem. Phys. Lett. $364(1-2)$ (2002) 139-143.

[49] T. Nakano, T. Kaminuma, T. Sato, Y. Akiyama, M. Uebayasi, K. Kazou, Fragment molecular orbital method application to polypeptides, Chem. Phys. Lett. 318 (6) (2000) 614-618.

[50] K. Kitaura, T. Sawai, T. Asada, T. Nakano, M. Uebayasi, Pair interaction molecular orbital method an approximate computational method for molecular interactions, Chem. Phys. Lett. 312 (1999) 319-324.

[51] R. Landauer, Irreversibility and heat generation in the computing process, Ibm J. Res. Dev. 44 (1.2) (2000) 261-269. 